\title{
Processing Optimization of High Speed Machining in Numerical Control Manufacturing
}

\author{
Jun $\mathrm{Wu}^{1, *}$ and Yuanxiang Zhang ${ }^{2}$ \\ ${ }^{1}$ College of Mechanical Engineering, Quzhou University, Quzhou, 324000, China \\ ${ }^{2}$ Jiuhuabei Road-78, Kecheng district, Quzhou City Country. Postcard: 324000, China
}

\begin{abstract}
High cost and low efficiency problem has been bothering NC teaching. To lower the cost of practical teaching of higher speed numerical control manufacturing along with improvement in processing efficiency, a multi-objective scheme for optimization of process parameters has been developed here. Various constraint conditions were set and a complex algorithm was employed for optimization. To achieve high production efficiency, lowering processing cost and comprehensive optimization, a mathematical model to calculate optimal cutting parameters with high accuracy is presented here. Specifically, basic elements for numerical control manufacturing and different types of cooling manufacturing were analyzed. Optimization of major parameters, such as cutting speed, radial cutting quantity and back engagement of cutting edge are discussed here. These parameters provide a reference for proper selection of processing parameters during machining. The feasibility of this scheme was validated by application in practical teaching, which improves processing efficiency and reduces cost of experimental materials.
\end{abstract}

Keywords: Optimization, experimental teaching, application, high speed machining.

\section{INTRODUCTION}

High Speed Machining (HSM) is widely employed in various applications such as mould manufacturing along with a wide variety of industries, which include aerospace, automobile, electronics and processing. HSM has various advantages such as high efficiency, high-precision, high surface quality, high hardness materials processing, low cost, low cutting force and low cutting temperature. As a result, it is gradually replacing abrasive and electro-processing methods. This processing mode uses high speed, high feed and low cutting quantity originated from the assumption proposed by the German cutting physical scientist Dr. Salomon in 1931, which states that at a certain cutting speed that exceeds a critical value, the chip/tool interface temperature will start to decrease along with the unit cutting force [1].

As theoretical research on HSM improves along with technical hardware breakthroughs such as the invention allowing the use of high speeds in various machine tools, cutting tools and $\mathrm{CAD} / \mathrm{CAM}$ processing software. The industrial implementation of HSM is substantially facilitated. However, determination of processing parameters has been a limiting factor for optimal operation of HSM processing. Therefore, the study of machining mechanism, the determination of optimal processing parameters and validation of optimization scheme have become among the key research topics in HSM.

\section{OPTIMIZATION STRATEGY OF HSM}

\subsection{Processing Programming Trategy}

Besides the requirement of non-interference and noncollision, the following processing notes should be strictly followed during HSM.

(1) Feed mode of cutting tool should be from the tangential direction, rather than the normal direction. The cutting speed should be kept as low as possible during the first cut, so it is in the cutting out of work piece.

(2) All tool paths should be continuous and smooth to prevent influence on cutting stability by sudden change of tool path.

(3) Cutting feed and cutting tool penetration should be evenly matches to allow for a longer tool service life and guarantee a smooth cutting load.

(4) Rough machining and fine machining should be conducted based on highest cutting efficiency and highest processing precision.

\subsection{Analysis of the Cutting Elements}

The determination of cutting speed $\mathrm{Vc}$, feed $\mathrm{f}$, radial cutting quantity ae, and axial cutting penetration ap (back engagement of cutting edge) directly affects surface processing quality of the work piece, production efficiency, and processing cost.

(1) Cutting speed is given as, 
$V_{C}=\frac{N \times \pi \times D}{1000} \mathrm{~m} / \mathrm{min}$

where, $N$ is the main shaft speed (for HSM, 15000r/min$40000 \mathrm{r} / \mathrm{min}$ ), $\mathrm{D}$ is effective diameter of cutting tool.

(2) Feed is given by the equation,

$V_{f}=N \times f_{\mathrm{Z}} \times Z$

where, $N$ is the speed of milling tool, $f_{\mathrm{Z}}$ is the feed to each tooth, $Z$ is the number of teeth.

The above formula indicates that, an increase in cutting speed and milling tool speed while keeping Vf constant, the feed to each tooth $f_{\mathrm{Z}}$ decreases. Furthermore, if $f_{\mathrm{Z}}$ is smaller than the arc radius of tool nose, the cutting process becomes more difficult, surface quality decreases and causes vibration Therefore, the optimization of $V_{f}$ is significantly related to cutting speed $V_{C}$.

(3) Radial cutting quantity ae, and back engagement of cutting edge $a_{p}$

When the radial cutting quantity ae or axial cutting penetration ap is smaller than the cutting tool radius, the actual cutting piece depth is smaller than the feed to each tooth. During the machining processing, the variation of ae, ap results in a continuous change of cutting penetration and material removal rate. It has been proved that both the cutting penetration and material removal rate are related to $f_{\mathrm{Z}}$. Therefore, optimizing $f_{z}$ would allow self-adjustment according to varying cutting conditions. This allows a constant cutting force and ensures machine stability during high speed processing.

\subsection{Analysis of Cooling Methods}

During high speed cutting process, scraps take away majority of the heat produced by the cutting tool. Therefore, the processing materials and selected cutting tools should be taken into consideration when choosing a proper cooling method. Following empirical results were obtained from experiments: 1) dry cutting should be employed to process aluminum alloy and cast-iron. 2) Air oil mist should be adopted when processing die steel. 3) Nitrogen oil mist should be used when processing titanium and nickel alloys with carbide cutter. 4) For high speed cutting conditions, liquid cooling method should not be used since the carbide cutter, which is usually used for high speed cutting, possesses high hardness but poor (missing word). As a result, it would easily break off on being cooled suddenly.

\section{OPTIMIZATION OF CUTTING ELEMENTS IN HSM}

\subsection{Development of Mathematical Model}

With an emphasis towards high quality, high production efficiency, and low cost, an optimal cutting quantity combination model is constructed. The cutting parameters are optimized through multi-objective comprehensive control. The details of the computational process are as follows.

\section{(1) The Highest Production Efficiency Principle}

According to reference [2], the unit time for each single cutting tool machining for one product is

$T_{A L L}\left(v, f_{z}\right)=t_{s}+t_{m}\left(v, f_{z}\right)+t_{c} \times \frac{t_{m}\left(v, f_{z}\right)}{t_{t}\left(v, f_{z}\right)}$

in which, $t_{s}$ is preparation time, $t_{m}$ is processing time, $t_{c}$ is turret indexing time, $t_{t}$ is the average lifetime of the cutting tool. According to reference [3]:

$t_{t}\left(v, f_{z}\right)=\left(\frac{C_{v} D q_{v}}{v f_{z} a_{p}^{x_{v}} a_{e}^{u_{v}} z^{p_{v}}} k_{v}\right)^{\frac{1}{m}}$

where, $C_{v}, q_{v}, X_{v}, U_{v}, P_{v}, k_{v}, m$ are all constant coefficients.

(2) The Lowest Processing Cost Principle

According to reference [2], the definition of single element processing cost is

$$
C_{P}\left(V, f_{z}\right)=T_{A L L}\left(v, f_{z}\right) \times H c+t_{m}\left(v, f_{z}\right) \times \frac{C_{t}}{t_{t}\left(v, f_{z}\right)}
$$

where, $C_{t}$ is the cutter cost, $H c$ is the cost of unit labor time.

\section{(3) Comprehensive Optimization Principle}

Multi-objective control function was constructed based on the multi-objective linear weight sum method to achieve the objective of simultaneously optimizing of processing cost and production efficiency. The function is as follows:

$$
M=\lambda_{1} \frac{T_{A L L}}{T_{A L L 0}}+\lambda_{2} \frac{C_{p}}{C_{p 0}}
$$

in which, $\lambda_{1}$ and $\lambda_{2}$ are weight coefficients, reflecting the significance of processing time and processing cost, which can be set by the process programming staff according to different requirements. $T_{A L L 0}$ and $C_{p 0}$ are the processing time and cost before optimization, respectively. They are regulated to eliminate the influence of processing time and cost on the optimization target.

\subsection{Development of Constraint Conditions}

Due to limitations of the processing equipments, cutting conditions and work piece quality, the machining parameters variables must be restricted.

\section{(1) Constraint on machine power}

The actual processing power $p$ must be smaller than the allowable processing power $P_{m}$. 


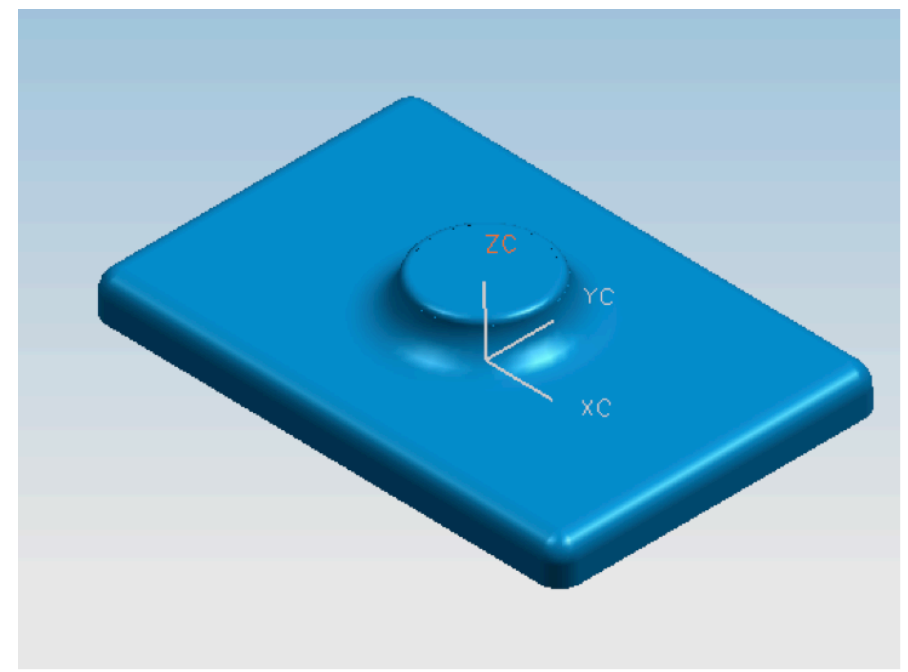

Fig. (1). Three-dimension of the convex mold model.

Table 1. Comparison of optimization results.

\begin{tabular}{|c|c|c|c|c|}
\hline \multirow{2}{*}{ Process } & \multicolumn{2}{|c|}{ Pre-optimization } & \multicolumn{2}{|c|}{ Post-optimization } \\
\cline { 2 - 5 } & Time/min & Cost/Yuan & Time/min & 30.5 \\
\hline \hline Rough machining & 40.2 & 92.9 & 32.1 & 71.5 \\
\hline Semi fine machining & 35.4 & 72.3 & 73.5 & 65.7 \\
\hline Fine machining & 136.7 & 375.6 & 203 \\
\hline
\end{tabular}

$p=\frac{F_{c} V_{c}}{6 \times 10^{4}}<e P_{m}$

where, $F_{c}$ is peripheral machining force, $V_{c}$ is machining speed, $e$ is the machine efficiency.

(2) Constraint on machine speed and feed

$V_{\min }<V<V_{\max } f_{\min }<f<f_{\max }$

(3) Constraint on surface roughness of work piece

$\mathrm{g}\left(\mathrm{x}_{1}, \mathrm{x}_{2}\right)=\mathrm{x}_{2}-\sqrt{R_{\max } / 8 r^{\varepsilon}} \leq 0$

in which, $R_{\max }$ is the minimum surface roughness, $R$ is milling tool radius.

\subsection{Algorithms}

In this paper, the complex algorithm is employed for the optimization, which is an important direct method for solving nonlinear constrained optimization problems. It does not require calculating gradient of objective function, but selecting simplex vertices and comparing each target function value at each vertex. Therefore, as one of the most optimal direct methods, the calculation is relatively simple $[4,5]$.

\section{OPTIMIZATION RESULTS FROM A PRACTICAL TEACHING OF NUMERICAL CONTROL}

The convex mold model of an oil reservoir port was built using UG4 (Fig. 1). Duralumin is selected as the raw material, and machine C1200U was chosen for manufacturing. A rated power of $\mathrm{P}=23 \mathrm{KW}$ and rotational speed $\mathrm{n}=5000 \sim 24000$ $\mathrm{r} / \mathrm{min}$ were used. The cutting tool is FEW, CARBIDE ENDMILL with $\mathrm{D}=10 \mathrm{~mm}$ and $\mathrm{r} \varepsilon=3 \mathrm{~mm}$. The processing requirement for the minimum surface roughness is $\mathrm{Ra}=3$. $2 \mu \mathrm{m}$.

General parameters are $\mathrm{K} 1=2.2 \mathrm{Yuan} / \min , \mathrm{Xv}=0.12$, $\mathrm{Yv}=0.63, \mathrm{Uv}=0.34, \mathrm{Pv}=0.11$ and $\mathrm{m}=0.36$. The above parameters were set in the calculation program, and the comparison table between processing time and cost was obtained after optimization and arrangement, as shown in Table 1.

\section{CONCLUSION}

In this paper, a practical teaching case of numerical control (convex mold model of an oil reservoir port) was selected as the optimization object with processing parameters. The feasibility of multi-objective processing parameters optimization control is demonstrated. The results show a reduction of the practice costs, and a saving in the teaching time, 
which provides a certain reference value for the research of HSM processing.

\section{CONFLICT OF INTEREST}

The authors confirm that this article content has no conflict of interest.

\section{ACKNOWLEDGEMENTS}

This work is supported by Quzhou Science and Technology Bureau Project of China (20121049 \& 20121048).

\section{REFERENCES}

[1] C. Y. Nian, W. H. Yang, Y. S. Tarng, "Optimization of turning operations with multiple performance characterisitics", J. Mat. Proces. Tech., vol. 95, pp. 90-96, 1999.

[2] H. Yau, M. Kuo, "NURBS machining and feedrate adjustment for high-speed cutting of complex sculptured", Int. J. Prod. Res., vol. 39, pp. 21-41, 2001.

[3] L. N. L. de Lacalle, A. Lamikiz, J. A. Sanchez, J. L. Arana, "Improving the surface finish in high speed milling of stamping dies", J. Mat. Proces. Tech., vol. 123, pp. 292-302, 2002.

[4] K. Morishige, T. Sakamoto, Y. Takeuchi, "Development of CAM system for high speed milling", In: Proc. $3^{\text {rd }}$ Int. Conf. Metal Cutt. High Speed Mach., Metz, 2001, pp.341-350.

[5] M. B. Yang, H. Li, H. Yang, Metal Material Experiment Manual, Beijin: Chemical Industry Press, 2007.

(C) Wu and Zhang; Licensee Bentham Open.

This is an open access article licensed under the terms of the Creative Commons Attribution Non-Commercial License (http://creativecommons.org/licenses/by-nc/3.0/) which permits unrestricted, non-commercial use, distribution and reproduction in any medium, provided the work is properly cited. 